\title{
FREE DENDRIFORM ALGEBRAS. PART I. A PARENTHESIS SETTING
}

\author{
PHILIPPE LEROUX
}

Received 25 September 2005; Revised 18 September 2006; Accepted 21 September 2006

We propose both a reformulation of some known results on the free dendriform algebra on one generator from a parenthesis setting instead of using permutations and some developments as well. Moreover, by introducing the concept of NCP-operad, we show how to use the free dendriform algebra on one generator to reformulate some results obtained by Speicher in free probability.

Copyright (c) 2006 Hindawi Publishing Corporation. All rights reserved.

\section{Introduction}

In Section 2, we propose a reformulation of the free dendriform algebra over the generator $Y$ via a parenthesis setting. We propose at the same time both a brief survey on trees and new results proved from the parenthesis setting. In Section 3, we present a bijection between planar rooted binary trees and noncrossing partitions. This allows the introduction of the concept of NCP-operads, whose axioms look like regular operads ones. We then show, in Section 4, how to use the free dendriform algebra on one generator to reformulate some results obtained by Speicher in free probability.

In this paper, $K$ is a characteristic zero field, $\mathbb{N}$ is the semiring of integers, and $\mathbb{N}_{b}^{n}$ stands for the set $\left\{\underline{v}:=\left(v_{1}, \ldots, v_{n}\right) \in \mathbb{N}^{n}\right.$; for all $\left.1 \leq i \leq n, 0<v_{i} \leq i\right\}$, in bijection with $S_{n}$, the symmetric group over $n$ elements. If $S$ is a finite set, then $\operatorname{card}(S)$ denotes its cardinal, $K S$, the $K$-vector space spanned by $S$, and $\langle S\rangle$, the free associative semigroup generated by $S$. Rooted planar binary trees will be called binary trees for short. For all $n>0$, we mean by $Y_{n}$ the set of planar rooted binary trees with $n+1$ leaves. If $k \in K, \underline{v} \in K^{n}$, then $(k+\underline{v})$ is the vector $\underline{v}$ whose coordinates have been shifted by $k$.

\section{Arithmetics on trees from operads}

For details about operads, the reader should consult the literature, for instance, $[1,4,10]$. Dendriform algebras have been introduced by Loday [5] as dual, in the operadic sense, to associative dialgebras, themselves motivated by $K$-theory. The free dendriform algebra on 
one generator is then closely related to binary trees. Major developments [7] have been put forward by using the Hopf algebra structure on the regular representations of the permutation groups founded by Malvenuto and Reutenauer [9] and connections between permutations and binary trees. Since then, an arithmetic on trees has been introduced by Loday [6]. The aim of this section is to present another way to handle the free dendriform algebra on one generator. Instead of starting with coding binary trees via permutations, we focus on the parenthesizing the meaning of binary trees.

2.1. Binary trees versus vectors. Contrary to permutations used in $[6,8]$, we propose in this section another way to encode binary trees compatible with the Tamari order which will play a key role to explicit operations on trees. For that, we associate with a planar binary tree of $Y_{n}$ a unique vector of $\mathbb{N}^{n}$ in the following way. To any binary tree $\tau$ corresponds a unique parenthesizsing, and therefore a unique monomial in $\left\langle x_{1}, \ldots, x_{n+1},(),\right\rangle$ and thus a unique monomial in $\left\langle x_{1}, \ldots, x_{n+1},(\rangle\right.$ obtained by forgetting all right parentheses. Proceeding this way, we obtain an injection: $\operatorname{Exp}: Y_{n} \rightarrow\left\langle x_{1}, \ldots, x_{n+1},(\rangle\right.$. In the sequel, to ease notation, the unique parenthesizing associated with the binary tree $\tau$ will be also represented by $\operatorname{Exp}(\tau)$ as in the following example:

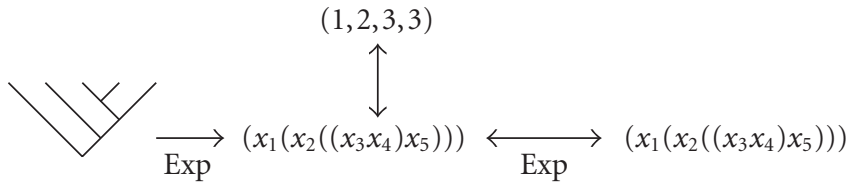

Encode the parentheses of $\operatorname{Exp}(\tau)$ of the binary tree $\tau$ in a vector $\underline{v}:=\left(v_{1}, v_{2}, \ldots, v_{n}\right)$ of $\mathbb{N}^{n}$ by declaring that for all $1 \leq i \leq n, v_{i}:=i$ if and only if there exists a left parenthesizing at the left-hand side of $x_{i}$, that is, ... $\left(p^{2} x_{i} \ldots\right.$, with $p>0$, occurs in the monomial $\operatorname{Exp}(\tau)$. Otherwise, there exists a unique rightmost parenthesis at the right-hand side of $x_{i}$ which closes a unique left parenthesis, say, open at $x_{j}$. In this case, $v_{i}:=j$. Observe that this framework works since binary trees, via their leaves, model all parentheses one can obtain from a binary operation. We then obtain an injective map; name : $Y_{n} \rightarrow \mathbb{N}^{n}$, which maps any tree $\tau$ into a vector, name $(\tau)$, also denoted by $\underline{\tau}$ for short, called the name of $\tau$. We warn the reader that we use the word "name" with a different meaning that in [6]. In the sequel, name $\left(Y_{n}\right)$ will be denoted by $\mathbb{A}^{n}$ (A), like Appellation), and by complete expression, we mean a monomial of $\left\langle x_{1}, \ldots, x_{n+1},(),\right\rangle$ in one-to-one correspondence with a rooted planar binary tree, that is, every parenthesis (is closed by a unique parenthesis ).

Proposition 2.1. Let $\underline{v} \in \mathbb{N}_{b}^{n}$.

There is a unique monomial ( ${ }^{q_{1}} x_{1}\left(q_{2} x_{2} \cdots\left({ }^{q_{n}} x_{n} x_{n+1}\right.\right.$ from $\left\langle x_{1}, \ldots, x_{n+1},(),\right\rangle$ associated with $\underline{v}$, where $q_{i}$ is the number of $i$ 's appearing in $\underline{v}$. Such an algorithm gives a surjective map Tree : $\mathbb{N}_{b}^{n} \rightarrow Y_{n}$.

Proof. We proceed by induction. Fix $\underline{v}:=\left(v_{1}, \ldots, v_{n}\right) \in \mathbb{N}_{b}^{n}$. Its associated monomial in $\left\langle x_{1}, \ldots, x_{n},(\rangle\right.$ is of the form $\left({ }^{q_{1}} x_{1}\left({ }^{q_{2}} x_{2} \cdots\left({ }^{q_{n}} x_{n} x_{n+1}\right.\right.\right.$, where $q_{j}$ is the number of $j$ 's appearing in $\underline{v}$. Observe that $\sum q_{j}=n$ and $1 \leq q_{j} \leq n-j+1$ since $j$ may appear only 
from $x_{j}$. Take the highest $j$, with $q_{j} \neq 0$, that is, consider $\left(q_{j} x_{j} x_{j+1} \cdots x_{n}\right.$. As parentheses model a binary operation, there is a unique way to set right parentheses, namely, $\left.\left(q_{j} x_{j} x_{j+1}\right) \cdots x_{j+q_{j}}\right) \cdots x_{n} x_{n+1}$. This gives a complete expression $\left.X=\left(q_{j} x_{j} x_{j+1}\right) \cdots x_{j+q_{j}}\right)$ and ${ }^{q_{1}} x_{1}\left(q_{2} x_{2} \cdots\left({ }^{q_{j-1}} x_{j-1} X \cdots x_{n+1}\right.\right.$ with $\sum q_{k}=n-q_{j}$. The proof is complete by induction.

Proposition 2.1 is in fact an error-correcting code. Let us apply it to $(1,2,1,2)$. This gives $\left(\left(x_{1}\left(\left(x_{2} x_{3} x_{4} x_{5}\right.\right.\right.\right.$, that is, $\left(\left(x_{1}\left(\left(x_{2} x_{3}\right) x_{4}\right)\right) x_{5}\right)$, that is, the tree named by $(1,2,2,1)$.

Corollary 2.2 (reconstruction criterion). A vector $\underline{v} \in \mathbb{N}_{b}^{n}$ is the name of a binary tree if and only if name $(\operatorname{Tree}(\underline{v}))=\underline{v}$.

2.1.1. Realization of the grafting operation. In the sequel, for all $n>0, \underline{n}:=(1,2,3,4, \ldots, n)$, $\underline{0}:=(0)$, and $\underline{1_{n}}:=(1,1,1, \ldots, 1)$. Fix $n, m \neq 0$ and $\underline{v} \in \mathbb{A}^{n}$ and $\underline{w} \in \mathbb{A}^{m}$. The grafting operation is a map,

$$
\begin{gathered}
\vee: \mathbb{A}^{n} \times \mathbb{A}^{m} \longrightarrow \mathbb{A}^{m+n+1}, \\
(\underline{v}, \underline{w}) \longmapsto \underline{v} \vee \underline{w}:=\left(\underline{v}, 1, w_{1}+n+1, \ldots, w_{m}+n+1\right):=(\underline{v}, 1, v+1+\underline{w}),
\end{gathered}
$$

where for all $k \in \mathbb{N}$ and $\underline{w} \in \mathbb{A}^{m}, m>0$, the notation $k+\underline{w}$ stands for $\left(w_{1}+k, \ldots, w_{m}+k\right)$, $k+\underline{0}:=\underline{0}$ and where by abuse of notation $v$ denotes the number of coordinates in $\underline{v}$ (i.e., $n$ in this case). In the sequel, we give the name (0) to the tree $\mid$ and (1) to the tree $Y:=Y$. By convention, if $\underline{v}:=\left(v_{1}, \ldots, v_{n}\right) \in \mathbb{A}^{n}$ and $n \neq 0$, then $\underline{v} \vee(0):=(\underline{v}, 1),(0) \vee \underline{v}:=(1,1+\underline{v})$, and $(0) \vee(0):=(1)$. There exists a trivial partial order in $\mathbb{N}^{n}$ by declaring that $\underline{v} \leq \underline{w} \Leftrightarrow$ for all $1 \leq i \leq n, v_{i} \leq w_{i}$, inducing so a trivial partial order on $\mathbb{A}^{n}$. As already mentioned, there exists a partial order on binary trees, often called the Tamari order, induced by the relation $\left(\tau_{1} \vee \tau_{2}\right) \vee \tau_{3} \leq \tau_{1} \vee\left(\tau_{2} \vee \tau_{3}\right)$, for any trees $\tau_{1}, \tau_{2}, \tau_{3}$. Equip $Y_{n}$ with the Tamari order. Then, for all $\pi, \tau \in Y_{n}, \pi<\tau$ if and only if name $(\pi)<$ name $(\tau)$. There is, on the symmetric group $S_{n}$, a partial order $\leq_{\text {Bruhat }}$ called the weak-Bruhat order. From [8], there is a surjective map, $s \mapsto Y_{s}$, mapping permutations of $S_{n}$ to $n$-trees of $Y_{n}$. Equipped with the Tamari order, it is proved that $s \leq_{\text {Bruhat }} s^{\prime} \Leftrightarrow Y_{s} \leq Y_{s}$. Therefore, the weak-Bruhat order of the symmetric group $S_{n}$ is nothing else that the trivial partial order on $\mathbb{A}^{n}$. As $S_{n}$ is in bijection with $\mathbb{N}_{b}^{n}$, it might be interesting to find an order preserving code between permutations and vectors of $\mathbb{N}_{b}^{n}$. The Tamari order is represented for $\left(Y_{2},<\right)$ or $\left(\mathbb{A}^{2},<\right)$, $Y(1,1) \rightarrow Y(1,2)$, and for $\left(Y_{3},<\right)$ or $\left(\mathbb{A}^{3},<\right)$, by

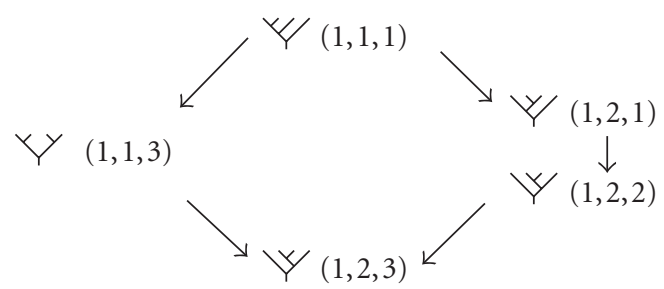


For these lattices, the Möbius function, $M$, can be explicited. For all $\vec{v}=\left(v_{1}, \ldots, v_{n}\right) \in \mathbb{A}^{n}$, $M(\vec{v}):=(-1)^{t_{\vec{v}}}$ if and only if for all $i, v_{i}=1$ or $i$, and where $t_{\vec{v}}$ is the number of coordinates such that $v_{i}=i \neq 1$. Else, $M(\vec{v}):=0$ [14]. We extend the Möbius function to $(0)$ by setting $M(\underline{v} \vee(0)):=M(\underline{v}, 1):=M(\underline{v})$, and $M((0) \vee \underline{v}):=M((1,1+\underline{v}):=0$, unless $\underline{v}=\underline{n}$, for all $\underline{v} \in \mathbb{A}^{n}$ and $n>0$.

Proposition 2.3. Let $\pi \in Y_{n}$ and $\tau \in Y_{m}$. Then, $\underline{\text { name }}(\pi \vee \tau)=\underline{\text { name }}(\pi) \vee \underline{\text { name }}(\tau)$. (The map name is a grafting morphism.) Moreover, $M(\underline{\text { name }}(\pi \vee \tau))=(-1)^{m} M(\underline{\text { name }}(\pi))$ if $\tau=\underline{m}$ and zero otherwise. Let $\underline{v}, \underline{w} \in\left(\mathbb{A}^{n},<\right)$ and $\underline{u}, \underline{z} \in\left(\mathbb{A}^{m},<\right)$. Then $(\underline{v} \leq \underline{w}, \underline{u}<\underline{z})$ or $(\underline{v}<\underline{w}, \underline{u} \leq \underline{z})$ if and only if $\underline{v} \vee \underline{u}<\underline{w} \vee \underline{z}$.

Proof. Let $\pi \in Y_{n}$ and $\tau \in Y_{m}$. The tree $\pi$ gives a unique complete expression, $\operatorname{Exp}(\pi)=$ ${ }^{p_{1}} x_{1}\left(p_{2} x_{2} \cdots\left({ }^{p_{n}} x_{n} x_{n+1}\right.\right.$, where $p_{i}$ is the number of $i$ 's in the name of $\pi$. Similarly for $\tau$, set $\operatorname{Exp}(\tau)=\left(p_{1}^{\prime} x_{1}\left(p_{2}^{\prime} x_{2} \cdots\left(p_{m}^{\prime} x_{m} x_{m+1}\right.\right.\right.$. Their grafting gives

$$
\left(\left({ } ^ { p _ { 1 } } x _ { 1 } \left({ } ^ { p _ { 2 } } x _ { 2 } \cdots \left({ } ^ { p _ { n } } x _ { n } x _ { n + 1 } \left({ } ^ { p _ { 1 } ^ { \prime } } x _ { 1 } \left({ } ^ { p _ { 2 } ^ { \prime } } x _ { 2 } \cdots \left({ }^{p_{m}^{\prime}} x_{m} x_{m+1},\right.\right.\right.\right.\right.\right.\right.
$$

which once renamed in a complete expression of $\left\langle x_{1}, \ldots, x_{n+1}, x_{n+2}, \ldots, x_{n+1+m+1},(\rangle\right.$ gives

$$
\left(\left({ } ^ { p _ { 1 } } x _ { 1 } \left({ } ^ { p _ { 2 } } x _ { 2 } \cdots \left({ } ^ { p _ { n } } x _ { n } x _ { n + 1 } \left({ } ^ { p _ { 1 } ^ { \prime } } x _ { n + 1 + 1 } \left({ } ^ { p _ { 2 } ^ { \prime } } x _ { n + 1 + 2 } \cdots \left({ }^{p_{m}^{\prime}} x_{n+1+m} x_{n+1+m+1} .\right.\right.\right.\right.\right.\right.\right.
$$

Observe that name $(\pi \vee \tau)_{n+1}=1$, giving the first claim. For computing the Möbius function, observe that if $1 \leq w_{i}<i$, then $n+2 \leq \underline{\text { name }}(\pi \vee \tau)_{i+n+1}<i+n+1$. Without forgetting $w_{1}=1$, which becomes name $(\pi \vee \tau)_{1+n+1}=n+2>1$, we obtain $M(\underline{\text { name }}(\pi \vee \underline{m}))=$ $(-1)^{m} M(\underline{\text { name }}(\pi))$ and zero otherwise. The last claim is straightforward.

The grafting operation can be extended by bilinearity to $K \mathbb{A}^{\infty}:=\bigoplus_{n \geq 0} K \mathbb{A}^{n}$. In the sequel, we set $K \mathbb{A}_{*}^{\infty}:=\bigoplus_{n \geq 1} K \mathbb{A}^{n}, \mathbb{A}^{\bullet}:=\bigcup_{n \geq 0} \mathbb{A}^{n}$, and $\mathbb{A}_{*}^{*}:=\bigcup_{n \geq 1} \mathbb{A}^{n}$.

2.1.2. Coding the over and under operations. Before going on, recall that an associative $L$-algebra is a $K$-vector space $A$ equipped with two binary operations $\nearrow, \nwarrow: A^{\otimes 2} \rightarrow A$ and obeying three constraints. The two operations are associative and verify $(x>y) \backslash z:=x \succ$ $(y \backslash z)$. L-monoids are straightforward to define. From a (co)algebraic point of view, $L$ coalgebras have been introduced on graphs in $[2,3]$. In [8], Loday and Ronco introduced the operations over and under on trees, denoted, respectively, by $\nearrow,\left\ulcorner: Y_{n} \times Y_{m} \rightarrow Y_{n+m}\right.$, for all $n, m \neq 0$, where $\pi \nearrow \tau$ is the tree $\tau$ with its leftmost leaf identified with the root of $\pi$ and where $\pi \wedge \tau$ is the tree $\pi$ with its rightmost leaf identified with the root of $\tau$. These two operations have a common unit which is $\mid$. To define the analogue of these two operations on vectors, consider the map $\triangleright: \mathbb{N} \times \mathbb{A}^{n} \rightarrow \mathbb{A}^{n}, k \triangleright \underline{v}:=\left(k \tilde{+} v_{1}, \ldots, k \tilde{+} v_{n}\right)$, where $k \tilde{+} v_{i}:=k+v_{i}$, for $v_{i} \neq 1$ and $k \tilde{+} 1:=1$ (otherwise stated, 1 is a right annihilator for the operation $\tilde{+}$ ).

Proposition 2.4. Fix $n, m \neq 0$ and $\underline{v} \in \mathbb{A}^{n}$ and $\underline{w} \in \mathbb{A}^{m}$. The binary operations $\nearrow, \backslash: \mathbb{A}^{n} \times$ $\mathbb{A}^{m} \rightarrow \mathbb{A}^{n+m}$ defined as follows: $\underline{v} \succ \underline{w}:=(\underline{v}, v \triangleright \underline{w})$ and $\underline{v} \backslash \underline{w}:=(\underline{v}, v+\underline{w})$, turn $\mathbb{A}_{*}^{*}$ (resp., $K \mathrm{~A}_{*}^{\infty}$ ) into an associative L-monoid (resp., an associative L-algebra). The map name is a morphism of associative L-monoids (resp., of associative L-algebras). Moreover, $M(\underline{v}\ulcorner\underline{w})=$ $M(\underline{v}) M(\underline{w})$ and $M(\underline{v} \backslash \underline{w})=(-1)^{m} M(\underline{v})$, if $\underline{w}=\underline{m}$, else 0 . 
Proof. Fix $n, m \neq 0$ and $\underline{v} \in \mathbb{A}^{n}$ and $\underline{w} \in \mathbb{A}^{m}$. Their complete expression $\operatorname{Exp}(\underline{v})$ (resp., $\operatorname{Exp}(\underline{w}))$ is of the form $\left({ }^{p_{1}} x_{1}\left(p_{2} x_{2} \cdots\left({ }^{p_{n}} x_{n} x_{n+1}\right.\right.\right.$ (resp., $\left(p_{1}^{\prime} x_{1}\left(p_{2}^{\prime} x_{2} \cdots\left(p_{m}^{\prime} x_{m} x_{m+1}\right)\right.\right.$. The associated trees are $\operatorname{Tree}(\underline{v})$ and $\operatorname{Tree}(\underline{w})$. However, $\operatorname{Tree}(\underline{v}) \nearrow \operatorname{Tree}(\underline{w})$ has the expression, $\left(p_{1}^{\prime} \operatorname{Exp}(\underline{v})\left(p_{2}^{\prime} x_{2} \cdots\left(p_{m}^{\prime} x_{m} x_{m+1}\right.\right.\right.$. Observe that $(\underline{v} \nearrow \underline{w})_{n+1}$ corresponds to $x_{n+1}$ thus is equal to 1 . Observe also that the left parentheses of $\operatorname{Exp}(\underline{w})$ do not move during this operation. We have to take into account the shift of the coordinate $j$ of $\underline{w}$ of an amount of $v$-corresponding to the degree of the tree Tree $(\underline{w})$ - for all $w_{j} \neq 1$. For $w_{j}=1$, the rightmost parenthesis at the right-hand side of $x_{j}$ still closes a left parenthesis at the left-hand side of $x_{1} \equiv \operatorname{Exp}(\underline{v})$. Therefore, for those $j,(\underline{v} \nearrow \underline{w})_{j}=1$. This gives the vector $\left(\underline{v}, 1, v \tilde{+} w_{2}, v \tilde{+} w_{3}, \ldots, v \tilde{+} w_{m}\right)=(\underline{v}, v \triangleright \underline{w})$. The second operation is easier since all the $w_{j}$ have to be shifted by $n=v$. We extend easily these two operations to $K \mathbb{A}_{*}^{\infty}$ by bilinearity. Observe then that $\nearrow$ and $\backslash$ are associative and the equality $\underline{u} \nearrow(\underline{v} \backslash \underline{w})=(\underline{u} \succ \underline{v}) \backslash \underline{w}$ holds, giving an associative $L$-monoidal structure to $\mathbb{A}_{*}^{*}$ or an associative $L$-algebra structure to $K \mathbb{A}_{*}^{\infty}$. Extend the map name by linearity, we obtain an isomorphism of associative $L$-algebras between $K Y_{*}^{\infty}$ and $K \mathbb{A}_{*}^{\infty}$. Concerning the identities on the Möbius function, observe that $M(\underline{v} \nearrow \underline{w})=M(\underline{v}) M(\underline{w})$ since $w_{i}=i \neq 1$ if and only if $(\underline{v} \nearrow \underline{w})_{i+v}=i+v$ and $v_{i}=1$ becomes $(\underline{v} \succ \underline{w})_{i+v}=1$. However $M(\underline{v} \backslash \underline{m})=(-1)^{m} M(\underline{v})$ since we have also to take into account $w_{1}=1$ becoming $(\underline{v} \succ \underline{w})_{1+v}=1+v$ and for $\underline{w} \neq \underline{m}, M(\underline{v} \backslash \underline{w})=0$.

Corollary 2.5. For all $\underline{u}_{1}, \underline{u}_{2} \in\left(\mathbb{A}^{n},<\right), \underline{v}_{1}, \underline{v}_{2} \in\left(\mathbb{A}^{m},<\right)$, and $\underline{w}_{1}, \underline{w}_{2} \in(\mathbb{A} P,<), \underline{u}_{1} \leq \underline{u}_{2}$, $\underline{w}_{1} \leq \underline{w}_{2}, \underline{v}_{1} \leq \underline{v}_{2} \Leftrightarrow \underline{u}_{1} \backslash \underline{v}_{1} \backslash \underline{w}_{1} \leq \underline{u}_{2} \nearrow \underline{v}_{2} \backslash \underline{w}_{2}$, where the presence of a strict inequality on the left-hand side induces a strict one in the right-hand side. Moreover, $\underline{v}>\underline{w} \leq \underline{v} \backslash \underline{w}$ holds.

Proof. The proof is complete by using Propositions 2.3 and 2.4.

Since $\underline{v}=\underline{v}_{l} \vee \underline{v}_{r}=\underline{v}_{l} \succ(1) \backslash \underline{v}_{r}$, it is straightforward to prove that the free $L$-algebra over one generator $x$ is isomorphic to $\left(K A_{*}^{\infty}, \nearrow,\ulcorner)\right.$ by mapping $x$ to the generator $(1)$ (see also [11]). By anticipating the ideas of Loday (explained in detail below), one can convert operations $\zeta, \nwarrow$ into set operations called $L$-additions denoted by $+_{\gamma},+_{\star}: \mathbb{A}^{n} \times$ $\mathbb{A}^{m} \rightarrow \mathbb{A}^{n+m}$ where $\underline{v}+<\underline{u}:=\underline{v} \backslash \underline{u}$ and $\underline{v}+, \underline{u}:=\underline{v} \succ \underline{u}$. These additions are associative and noncommutative. Similarly, there is a notion of $L$-multiplication. As $\left(K \mathbb{A}_{*}^{\infty}, \nearrow, \triangleleft\right)$ is the free $L$-algebra on the generator (1), one can uniquely write any name of binary trees via only the operations $\nearrow$ and $\backslash$ and (1). Such a formula, for a vector $\underline{v}$, is called its universal expression and is denoted by $\Phi_{\underline{v}}((1))$, obtained by the following induction $\Phi_{\underline{v}}((1)):=$ ${\omega_{\underline{\underline{v}}}}((1)) \nearrow(1) \backslash{\omega_{\underline{\underline{v}}}}_{r}((1))$. For instance, $(1,1,3)=(1) \nearrow(1) \backslash(1)=\omega_{(1,1,3)}((1))$. The $L-$ multiplication of $\underline{u} \in \mathbb{A}^{n}$ by $\underline{v} \in \mathbb{A}^{m}$ is by definition $\underline{u} \widetilde{\widetilde{x}} \underline{v}:=\Phi_{\underline{u}}(\underline{v}) \in \mathbb{A}^{n m}$. For instance, $(1,1,3) \tilde{\ltimes} \underline{v}=(\underline{v}) \nearrow(\underline{v}) \backslash(\underline{v})$. Therefore, any name of $\mathbb{A}^{m}$, where $m$ is a prime number, will be prime for the $L$-arithmetics. Consider now the $K$-vector space $K[X]_{L}$ spanned by $\left\{X^{\underline{v}}, \underline{v} \in\left(\mathbb{A}_{*}^{*},+_{\gamma},+_{\downarrow}, \tilde{\ltimes}\right)\right\}$. This is the free $L$-algebra over the generator $X^{(1)}$ where, as expected, operations are defined by $X^{\underline{u}} \nearrow X^{\underline{v}}:=X^{\underline{u}}, \underline{v}, X^{\underline{u}} \backslash X^{\underline{v}}:=X^{\underline{u}+} \underline{v}$, and $\left(X^{\underline{u}}\right)^{\underline{v}}:=$ $X_{\underline{u}} \widetilde{\widetilde{v}}$, imitating the usual ploynomial algebra on one variable endowed with the usual arithmetics over $\mathbb{N}$. There is also a dendriform involution $\dagger$, described in Section 2.2.2. We summarize our investigation by the following theorem. 
Theorem 2.6. The set $\mathbb{A}_{*}^{*}$ equipped with the L-additions, $+_{>}$, and $+_{\star}$, and with the dendriform involution $\dagger$ is an involutive-graded L-monoid. The L-multiplication, $\tilde{\ltimes}$, is left distributive associative though noncommutative. For any names of trees, $\underline{u}, \underline{v},(\underline{u}+, \underline{v})^{\dagger}=$ $\underline{v}^{\dagger}+\ltimes \underline{u}^{\dagger},(\underline{u}+\vee \underline{v})^{\dagger}=\underline{v}^{\dagger}+, \underline{u}^{\dagger}$, and $(\underline{u} \tilde{\times} \underline{v})^{\dagger}=\underline{u}^{\dagger} \tilde{\ltimes} \underline{v}^{\dagger}$ hold. Moreover, equipped with the dendriform involution, the $K$-vector space $K[X]_{L}$ spanned by $\left\{X^{\underline{v}}, \underline{v} \in\left(\mathbb{A}_{*}^{*},+_{,},+_{\ltimes}, \tilde{\ltimes}\right)\right\}$ is the free involutive associative L-algebra over the generator $X^{(1)}$.

Proposition 2.7. Fix $n, m \neq 0$ and $\underline{x}, y \in\left(\mathbb{A}^{n},<\right)$ and $\underline{a}, \underline{b} \in\left(\mathbb{A}^{m},<\right)$. With regards to the trivial partial order, the map $\omega_{\underline{x}}: \mathbb{A}^{m} \rightarrow \mathbb{A}^{n m}$ is a lattice morphism, that is, $\underline{x} \tilde{\times} \underline{a}<\underline{x} \tilde{\tilde{x}} \underline{b} \Leftrightarrow$ $\underline{a}<\underline{b}$ and the map $\tilde{\widetilde{\ltimes}} \underline{a}: \mathbb{A}^{n} \rightarrow \mathbb{A}^{\underline{n} m}$ is also a lattice morphism, that is, $\underline{x} \tilde{\tilde{}} \underline{a}<\underline{y} \tilde{\sim} \underline{a} \Leftrightarrow \underline{x}<\underline{y}$.

Proof. Keep notation of Proposition 2.7 For the first claim, proceed by induction. It is true for $\underline{x}=(1)$, for $\underline{x}=(1,1)$ and for $\underline{x}=(1,2)$. By Corollary 2.5, $\underline{x} \tilde{\tilde{x}} \underline{a}<\underline{x} \tilde{\widetilde{\alpha}} \underline{b} \Leftrightarrow \underline{x}_{l} \tilde{\ltimes} \underline{a}+$,

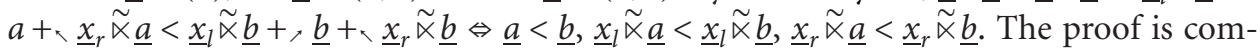
plete by induction. Concerning the second claim, if $\underline{x}<y$, then there exist say $k$ Tamari moves between the trees associated with $\underline{x}$ and $\underline{y}$. Suppose $k=1$. Then, in the definitions of $\underline{x}, y$, this means the existence of three vectors say $\underline{v}_{1}, \underline{v}_{2}, \underline{v}_{3}$ such that we have $\cdots\left(\underline{v}_{1} \vee \underline{v}_{2}\right) \vee \underline{v}_{3} \cdots<\cdots \underline{v}_{1} \vee\left(\underline{v}_{2} \vee \underline{v}_{3}\right) \ldots$ Therefore, we obtain, $\cdots\left(\underline{v}_{1} \nearrow(1) \backslash \underline{v}_{2}\right) \nearrow$ $(1) \backslash \underline{v}_{3} \cdots<\cdots \underline{v}_{1}>(1) \backslash\left(\underline{v}_{2}>(1) \backslash \underline{v}_{3}\right) \ldots$ The second claim holds for $k=1$, and thus for all $k$.

The operations $\nearrow, \backslash$ have a common unit which is $(0) \equiv \mid$. However, the link axiom of $L$-algebras is not compatible with this unit since it forces $\nearrow=\nwarrow$. Using the trivial partial order, we will exhibit an associative operation, sum of two nonassociative operations obeying three axioms. This operation has first been introduced by Loday and Ronco by using techniques in permutation groups [8]. One of the main advantages of our coding is to give easier proofs to these results.

Proposition 2.8. The following binary operation: $\star: K \mathbb{A}_{*}^{\infty} \otimes K \mathbb{A}_{*}^{\infty} \rightarrow K \mathbb{A}_{*}^{\infty}, \underline{v} \otimes \underline{w} \mapsto \underline{v} \star$ $\underline{w}=\sum_{\underline{v} \underline{w}^{\prime} \leq \underline{t} \leq \underline{v} \underline{v}^{\vee} \underline{t}} \underline{t}$, is associative. Moreover, $\underline{u} \star \underline{v} \star \underline{w}=\sum_{\underline{u}{ }^{\prime} \underline{v} \underline{w}^{\prime} \leq \underline{t} \leq \underline{u} \underline{u}^{\vee} \underline{v}^{\vee} \underline{w}} \underline{t}$ and $\underline{v} \star(0)=$ $\underline{v}=(0) \star \underline{v}$ hold for all $\underline{u}, \underline{v}, \underline{w} \in K \mathbb{A}_{*}^{\infty}$.

Proof. Let $\underline{u} \in \mathbb{A}^{p}, \underline{v} \in \mathbb{A}^{n}$, and $\underline{w} \in \mathbb{A}^{m}$, with $p, n, m \neq 0$. On the one hand, $\underline{u} \star(\underline{v} \star \underline{w})=$ $\sum_{\underline{u} \gamma \underline{v} \leq \underline{a} \leq \underline{u} \backslash \underline{v} \underline{a}} \star \underline{w}=\sum_{\underline{b} \in J_{1}} \underline{b}$, where $J_{1}:=\{\underline{b} ; \underline{a} \nearrow \underline{w} \leq \underline{b} \leq \underline{a} \backslash \underline{w}, \underline{u} \nearrow \underline{v} \leq \underline{a} \leq \underline{u} \backslash \underline{v}\}=$ $\{\underline{b} ;(\underline{u}, u \triangleright \underline{v},(u+v) \triangleright \underline{w}) \leq \underline{b} \leq(\underline{u}, u+\underline{v},(u+v)+\underline{w})\}$. On the other hand $(\underline{u} \star \underline{v}) \star$ $\underline{w}=\sum_{\underline{v} \gamma \underline{w} \leq \underline{c} \leq \underline{v} \backslash \underline{w} \underline{u}} \star \underline{c}=\sum_{\underline{d} \in J_{2}} \underline{d}$, where $J_{2}:=\{\underline{d}, \underline{u} \nearrow \underline{c} \leq \underline{d} \leq \underline{u} \backslash \underline{c}, \underline{v} \nearrow \underline{w} \leq \underline{c} \leq \underline{v} \backslash \underline{w}\}=$ $\{\underline{d},(\underline{u}, u \triangleright(\underline{v} \succ \underline{w})) \leq \underline{d} \leq(\underline{u}, u+(\underline{v} \backslash \underline{w}))\}=\{\underline{d},(\underline{u}, u \triangleright \underline{v},(u+v) \triangleright \underline{w}) \leq \underline{d} \leq(\underline{u}, u+$ $\underline{v},(u+v)+\underline{w})\}$. Hence $J_{1}=J_{2}$ and $\star$ is associative. The last claim is obvious since $(0)$ is by definition a unit for the operations $\nearrow$ and $\nwarrow$.

The sum in the definition of the associative product $\star$ can be split into two parts corresponding to two operations.

Proposition 2.9. Let $\underline{v}$ and $\underline{w}$ be names of some trees. Then, the set $I:=\{\underline{u} ; \underline{v} \nearrow \underline{w} \leq \underline{u} \leq$ $\underline{v} \backslash \underline{w}\}$ splits into two disjoint subsets: $I_{1}:=\left\{\underline{u} ;\left(\underline{v}, v_{l}+1+v_{r} \triangleright \underline{w}\right) \leq \underline{u} \leq(\underline{v}, v+\underline{w})\right\}$ and $\bar{I}_{2}:=\left\{\underline{u} ;(\underline{v}, v \triangleright \underline{w}) \leq \underline{u} \leq\left(\underline{v}, v+\underline{w}_{l}, 1, v+1+w_{l}+\underline{w}_{r}\right)\right\}$. 
Proof. First of all, observe that $(\underline{v}, v \triangleright \underline{w})=\left(\underline{v}, v \triangleright \underline{w}_{l}, 1, v+1+w_{l}+\underline{w}_{r}\right)$. Therefore, we have only to compare $\left(v \triangleright \underline{w}_{l}, 1\right)$ and $\left(v+\underline{w}_{l}, 1\right)$ in $I_{2}$. Similarly, concerning $I_{1}$, observe that $\left(\underline{v}, v_{l}+1+v_{r} \triangleright \underline{w}\right)=\left(\underline{v}, v_{l}+1+v_{r} \triangleright \underline{w}_{l}, v_{l}+2, v_{l}+1+v_{r} \triangleright\left[\underline{w}_{r}+w_{l}+1\right]\right)=$ $\left(\underline{v}, v_{l}+1+v_{r} \triangleright \underline{w}_{l}, v_{l}+2, v+1+w_{l}+\underline{w}_{r}\right)$ and $(\underline{v}, v+\underline{w})=\left(\underline{v}, v+\underline{w}_{l}, v+1, v+w_{l}+\right.$ $\left.1+\underline{w}_{r}\right)$. Therefore, we have to compare the vector $\left(v_{l}+1+v_{r} \triangleright \underline{w}_{l}, v_{l}+2\right)$ with $\left(v+\underline{w}_{l}\right.$, $v+1)$. As $\underline{v}_{l}$ represents a complete expression, jumps of coordinates situated after $v_{l}$ cannot take values below $v_{l}$. From this remark, one obtains that $I_{1}$ and $I_{2}$ are disjoint and $I_{1} \cup I_{2}=I$.

We recover from a vectorial framework the dendriform algebra introduced in [5]. Recall that a $K$-vector space $E$ is a dendriform algebra [5] if it is equipped with 2 binary operations $\prec$ and $\succ$ satisfying the following axioms for all $x, y \in E$ :

$$
(x \prec y) \prec z=x \prec(y \star z), \quad(x \succ y) \prec z=x \succ(y \prec z), \quad(x \star y) \succ z=x \succ(y \succ z),
$$

where, by definition, $x \star y:=x \prec y+x \succ y$, for all $x, y \in E$, where $\star$ turns out to be associative. We now adapt the following theorem appearing in $[5,8]$.

Theorem 2.10. Equip KA $\mathbb{A}_{*}^{\infty}$ with two binary operations $\prec$ and $\succ$, defined as follows: $\underline{v} \prec$ $\underline{w}:=\underline{v}_{l} \vee\left(\underline{v}_{r} \star \underline{w}\right)$ and $\underline{v} \succ \underline{w}:=\left(\underline{\nu} \star \underline{w}_{l}\right) \vee \underline{w}_{r}$, for all $\underline{v}, \underline{w} \neq(0)$. Then, $\left(K \mathbb{A}_{*}^{\infty}, \prec, \succ\right)$ is a dendriform algebra generated by (1). This space can be augmented by requiring $\underline{v} \prec(0):=$ $\underline{v}=:(0) \succ \underline{v}$ and $\underline{v} \succ(0):=0=:(0) \prec \underline{v}$, for $\underline{v} \neq(0)$. Equipped with these operations, $K \mathbb{A}^{\infty}$ is still a dendriform algebra with $\underline{v} *(0)=(0) * \underline{v}=\underline{v}$, for all $\underline{v} \in K \mathbb{A}^{\infty}$.

Theorem 2.11 (Loday [5]). The $K$-vector space $\left(K \mathbb{A}_{*}^{\infty}, \prec, \succ\right)$ is the free dendriform algebra on the generator (1).

As a corollary, there exists a universal expression, denoted by $\omega_{\underline{v}}(1)$, of $\underline{v} \in \mathbb{A}^{n}$ as a composition of $n$ copies of (1) with $\prec$ and $\succ$. Set $\omega_{(0)}(1):=0$ and of course $\omega_{\underline{v}}(1):=$ $\omega_{\underline{v}_{l}}(1) \succ(1) \prec \omega_{\underline{\underline{v}}_{r}}(1)$. For instance, $\omega_{(121)}(1):=((1) \prec(1)) \succ(1)$. So defined, $\left(K \mathbb{A}_{*}^{\infty}:=\right.$ $\left.\otimes_{n>0} K \mathbb{A}^{n}, \prec, \succ, \nearrow, \succ\right)$ is another presentation of the free dendriform algebra on one generator (1), equipped with an extra-structure of associative $L$-algebra $\left(K \mathbb{A}^{\infty}, \nearrow, \nwarrow\right)$ whose basis encodes binary trees in a compatible way with the Tamari order underlying the definitions of the operations $\prec$ and $\succ$. One of the main advantages of this coding lies in a reformulation of arithmetree in terms of vectors.

2.2. Arithmetree on planar binary trees. After the reformulations of constructions developed in [5, 8], let us recall a deep notion introduced by Loday. We follow [6]. A grove is simply a nonempty subset of $Y_{n}$, that is, a disjoint union of binary trees with same degree such that each tree appears only once. The set of groves over $Y_{n}$ is denoted by $\Psi_{n}$ and is of cardinal $2^{c_{n}}-1$. For instance, in low degrees,

$$
\mathbb{Y}_{0}:=\{\mid\}, \quad \mathbb{Y}_{1}:=\{Y\}, \quad \mathbb{Y}_{2}:=\{Y, Y, Y \cup Y\}
$$


Similarly, we define $\mathbf{A}^{n}$ in the same way. Instead of binary trees, we work with the set $\mathbf{A}^{n}$, which are the names of groves of $\mathbb{Y}_{n}$. Hence, $\mathbf{A}^{0}:=\{(0)\}, \mathbf{A}^{1}:=\{(1)\}, \mathbf{A}^{2}:=\{(1,1),(1,2)$, $(1,1) \cup(1,2)\}$, and continue to call grove such a union of vectors. The idea is now to convert the associative operation $\star$ in Proposition 2.8 into an addition with values in groves.

\subsubsection{The dendriform addition.}

Definition 2.12 (dendriform addition [6]). The dendriform addition of two vectors $\underline{v}$ and $\underline{w}$ associated with some planar binary trees is defined by

$$
\underline{v} \dot{+} \underline{w}:=\bigcup_{\underline{v} \underline{w}^{\prime} \leq \underline{u} \leq \underline{v^{\vee}} \underline{w}} \underline{u} .
$$

For instance, $(1) \dot{+}(1):=(1,1) \cup(1,2)$ or at the level of binary trees $Y \dot{+} Y=Y \cup Y$. This is extended to groves by distributivity of both sides, that is, $\bigcup_{i} \underline{v}_{i} \dot{+} \bigcup_{j} \underline{w}_{j}:=\bigcup_{i j}\left(\underline{v}_{i} \dot{+}\right.$ $\underline{w}_{j}$ ). Theorem 2.14 below proves that this definition has a meaning. This fundamental result has been found by Loday by using the following lemma whose proof can be simplified by using our vector codes.

Lemma 2.13. Let $\underline{w} \in \mathbb{A}^{n+m}$. Then, there exists unique $\underline{u} \in \mathbb{A}^{n}$ and $\underline{v} \in \mathbb{A}^{m}$ such that

$$
\underline{u} \nearrow \underline{v} \leq \underline{w} \leq \underline{u} \backslash \underline{v} .
$$

Proof (cf., to [6], Proposition 2.3, and Corollary 2.4). Recall that for $\underline{u} \in \mathbb{A}^{n}$ and $\underline{v} \in \mathbb{A}^{m}$, we get $\underline{u} \nearrow \underline{v}=(\underline{u}, u \triangleright \underline{v})$ and $\underline{u} \backslash \underline{v}=(\underline{u}, u+\underline{v})$. Take the first $n$ coordinates of $\underline{w} \in \mathbb{A}^{n+m}$. This gives a unique vector $\underline{u} \in \mathbb{A}^{n}$ according to Proposition 2.1. Consider the vector $\underline{v_{1}}$ defined by $\underline{v_{1}}:=\left(w_{n+1}, \ldots, w_{n+m}\right)$. Make the translation of $-n:=-u$ to obtain $\underline{v_{1}}-u=$ $\left(w_{n+1}-u, \ldots, w_{n+m}-u\right)$. The vector $\underline{v} \in \mathbb{A}^{m}$ we are looking for is obtained by replacing all negative or null coordinates by 1 . Observe that $\underline{u} \nearrow \underline{v}=(\underline{u}, u \triangleright \underline{v}) \leq \underline{w} \leq(\underline{u}, u+\underline{v})=$ $\underline{u} \backslash \underline{v}$.

Theorem 2.14 (Loday [6]). The dendriform addition of two groves is still a grove, that is, $\dot{+}: \mathbf{A}^{n} \times \mathbf{A}^{m} \rightarrow \mathbf{A}^{n+m}$.

Proposition 2.15 (left and right cancellations). Let $\underline{u}, \underline{v} \in \mathbb{A}^{m}, \underline{w} \in \mathbb{A}^{n}$. Then, $\underline{u} \dot{+} \underline{v}=$ $\underline{u} \dot{+} \underline{w} \Leftrightarrow \underline{v}=\underline{w}$ and $\underline{v} \dot{+} \underline{u}=\underline{w} \dot{+} \underline{u} \Leftrightarrow \underline{v}=\underline{w}$.

Proof. Using Proposition 2.4, recall that $\underline{u} \backslash \underline{v}=(\underline{u} ; u+\underline{v})$ and $\underline{u} \backslash \underline{w}=(\underline{u} ; u+\underline{w})$. The equality $\underline{u} \dot{+} \underline{v}=\underline{u} \dot{+} \underline{w}$ entails that $\underline{u} \backslash \underline{v}=\underline{u} \backslash \underline{w}$; hence $\underline{v}=\underline{w}$. The second equality is straightforward.

2.2.2. The dendriform involution. There is an involution on $\mathbf{A}^{\bullet}:=\bigcup_{n \geq 0} \mathbf{A}^{n}$ denoted by $\dagger$ and defined by $(\underline{v} \vee \underline{w})^{\dagger}:=\underline{w}^{\dagger} \vee \underline{v}^{\dagger}$. That is, $(\underline{v}, 1, v+\underline{w})^{\dagger}:=\left(\underline{w}^{\dagger}, 1, w+\underline{v}^{\dagger}\right)$. Doing so, observe that $(\underline{v}>\underline{w})^{\dagger}=\underline{w}^{\dagger} \backslash \underline{v}^{\dagger}$ and $(\underline{v} \backslash \underline{w})^{\dagger}=\underline{w}^{\dagger}>\underline{v}^{\dagger}$. Therefore, $(\underline{v} \dot{+} \underline{w})^{\dagger}=\underline{w}^{\dagger} \dot{+}$ $\underline{v}^{\dagger}$, that is, $\left(\mathbf{A}^{\bullet}, \dot{+}, \dagger\right)$ is an involutive graded monoid. Observe that $(1)^{\dagger}=(1)$ and by convention, we set $(0)^{\dagger}:=(0)$. We now state some properties of the involution on trees.

Proposition 2.16. Fix $n \geq 1$ and let $\operatorname{Inv}[n]:=\left\{\underline{v} \in \mathbb{A}^{n}, \underline{v}:=\underline{v}^{\dagger}\right\}$. Then, $\operatorname{Inv}[2 n]=\varnothing$ and $\operatorname{card}(\operatorname{Inv}[2 n+1])=c_{n}:=(1 /(n+1))\left(\begin{array}{c}2 n \\ n\end{array}\right)$. 
Proof. Observe that $\underline{v}=\underline{v}^{\dagger}$ if and only if there exists a unique $\underline{w}$ such that $\underline{v}=\underline{w} \vee \underline{w} \underline{w}^{\dagger}$.

[Trick to name $\left.\underline{v}^{\dagger}\right]$. Fix $\underline{v} \in \mathbb{A}^{n}$. There exists a very simple way to name $\underline{v}^{\dagger}$. Associate with $\underline{v}$, its complete expression in $\left\langle x_{1}, \ldots, x_{n}, x_{n+1},(),\right\rangle$. Relabel $x_{n+1}$ by $x_{1}, x_{n}$ by $x_{2}$ and so on. Read therefore from left to right such a monomial. The vector $\underline{v}^{\dagger} \in \mathbb{A}^{n}$ is obtained from the following construction. The coordinate $v_{i}^{\dagger}:=i$, for all $1 \leq i \leq n$, if and only if there is a ) at the right-hand side of $x_{i}$ and $v_{i}^{\dagger}:=j$ if the leftmost parenthesis ( at the left-hand side of $x_{i}$ closes a ) open in $x_{j}$. This works since the involution on binary trees is a symmetry with regards to the root axis, which can also be viewed as a symmetry with regards to an axis perpendicular to it — the Mirror axis—giving then the mirror image of the tree and thus its involution:

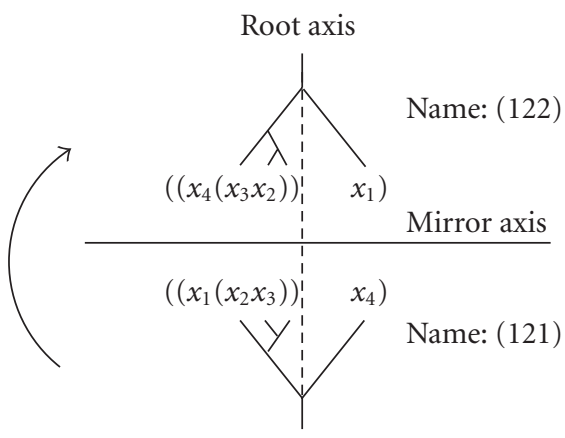

Proposition 2.17 (lattice anti-automorphism). Let $\underline{v}, \underline{w} \in \mathbb{A}^{n}$. Then, the dendriform involution is a lattice anti-automorphism, that is, $\underline{v}<\underline{w} \Leftrightarrow \underline{w}^{\dagger}<\underline{v}^{\dagger}$. Consequently, $M(\underline{v}, \underline{w})=$ $M\left(\underline{w}^{\dagger}, \underline{v}^{\dagger}\right)$, for any names of trees.

Proof. Fix $\underline{v}, \underline{w} \in \mathbb{A}^{n}$ with $\underline{v}<\underline{w}$. We will check the case when both $v_{i}=i=w_{i}$. In this case $v_{n+1-i}^{\dagger}=j$ and $w_{n+1-i}^{\dagger}=j^{\prime}$ with $j^{\prime} \leq j<N+1-i$. Indeed, suppose the existence of a ), the most external parenthesis standing at the right-hand side of $x_{j^{\prime}}$ and closing one ( open in $x_{i}$ in the complete expression associated with $\underline{w}$. As $\underline{v}<\underline{w}$, we get $v_{j^{\prime}} \leq w_{j^{\prime}}=i$. If $v_{j^{\prime}}=k<i$, then this means that the most external parenthesis ) standing at the righthand side of $x_{j^{\prime}}$ in the complete expression associated with $\underline{v}$ closes one (open in $x_{k}$. This implies that $v_{n+1-i}^{\dagger} \leq w_{n+1-i}^{\dagger}$. Checking every possibility leads to the conclusion that $v_{i}^{\dagger} \leq w_{i}^{\dagger}$ for all $1 \leq i \leq n$. The proof is complete since the dendriform involution is an involution. For the last claim, recall that the dual lattice of $\left(\mathbb{A}^{n},<\right)$ with order $\leq^{*}$ is defined such that $\underline{v} \leq^{*} \underline{w} \Leftrightarrow \underline{w} \leq \underline{v}$. Therefore, $\underline{v} \leq^{*} \underline{w} \Leftrightarrow \underline{v}^{\dagger} \leq \underline{w}^{\dagger}$, for any vectors of $\mathbb{A}^{n}$. The last claim holds since $M^{*}(\underline{v}, \underline{w})=M(\underline{w}, \underline{v})$ (see [13]).

2.2.3. The dendriform multiplication. The following idea developed by Loday consists in replacing the polynomial ring $K[X]$ (basis $\left(X^{n}\right)_{n \in \mathbb{N}}$ ) and well-known equations $X^{n} X^{m}:=$ $X^{n+m}$ and $\left(X^{n}\right)^{m}:=X^{n m}$ related to the usual arithmetic on $\mathbb{N}$ by planar binary trees. Instead of writing $K[X]$, one could have chosen $K[\mathbb{N}]$ to denote this polynomial ring. Consider the $K$-vector space $K\left[\mathbf{A}_{*}^{*}\right]$ spanned by the basis $\left\{X^{\underline{v}}, \underline{v} \in \mathbb{A}_{*}^{*}\right\}$. The space $K\left[\mathbf{A}_{*}^{*}\right]$ has a natural dendriform algebraic structure given by: $X^{\underline{u}} \prec X^{\underline{v}}:=X^{\underline{u}} \stackrel{-v}{v}$ and $X^{\underline{u}} \succ X^{\underline{v}}:=X^{\underline{u}} \vdash \underline{v}$, 
with the convention $X^{\underline{u} \cup \underline{v}}:=X^{\underline{u}}+X^{\underline{v}}$. As expected, $X^{\underline{u}} \star X^{\underline{v}}:=X^{\underline{u}} \dot{+} \underline{v}$, where $\star$ is the associative product, sum of $\prec$ and $\succ$. This nonunital associative algebra, another presentation of the free dendriform algebra on one generator, here $X^{(1)}$, can be augmented by adding the unit $1:=X^{(0)}$ so that, $K\left[\mathbf{A}^{\bullet}\right]:=K\left[\mathbf{A}_{*}^{*}\right] \oplus K \cdot 1$. By convention, we set $X^{\varnothing}=0$. As usual, the operations $\prec$ and $\succ$ can be partially extended to $K\left[\mathbf{A}^{\bullet}\right]$ by declaring that $1 \succ X^{\underline{v}}:=X^{\underline{v}}=: X^{\underline{v}} \prec 1$, for $\underline{v} \neq(0)$ and vanish otherwise, explaining the presence of the empty set. For instance, $1 \prec X^{\underline{v}}:=X^{(0)-\underline{v}}:=X^{\varnothing}:=0$, as expected. The notation $K[\mathbb{N}]:=K[X]$ stands for the usual polynomial algebra on one variable say $X$. As $X^{n} X^{m}:=X^{n+m}$ and $\mathbb{N}$ is invariant by addition, one can use also the notation $K[\mathbb{N}]$ without any ambiguity. However, $K\left[\mathbb{A}^{\bullet}\right]$ is not invariant by the dendriform addition, that is why we choose the notation $K\left[\mathbf{A}^{\bullet}\right]$ and not $K\left[\mathbb{A}^{\bullet}\right]$. The notation $K\left[\mathbf{A}^{\bullet}\right]$ stands for the $K$-vector space spanned by $\left\{X^{\underline{v}}, \underline{v} \in \mathbb{A}^{\bullet}\right\}$.

Definition 2.18 (dendriform multiplication [6]). The dendriform multiplication $\ltimes: \mathbf{A}^{n} \times$ $\mathbf{A}^{m} \rightarrow \mathbf{A}^{n m}$ is given by $\underline{u} \ltimes \underline{v}:=\omega_{\underline{u}}(\underline{v})$, for all $\underline{u}$ and $\underline{v}$ are names of binary trees and extended to groves via distributivity on the left with respect to the disjoint union, that is, $(\underline{u} \cup \underline{v}) \ltimes \underline{w}:=\underline{u} \ltimes \underline{w} \cup \underline{v} \ltimes \underline{w}$.

For instance, as $(1,2)=(1) \dashv(1)$, we get $(1,2) \ltimes \underline{v}=(\underline{v}) \dashv(\underline{v})$. Therefore, $(1,2) \ltimes$ $(1,1)=(1,1) \dashv(1,1)=(1,1,3,3)$. The dendriform multiplication is associative, not commutative, distributive on the left with regards to that the dendriform addition $\dot{+}$ has the neutral element (1) and is compatible with the involution $\dagger,(\underline{u} \ltimes \underline{v})^{\dagger}=\underline{u}^{\dagger} \ltimes \underline{v}^{\dagger}$. Moreover, the neutral element for $\dot{+}$, that is, $(0)$, is by convention a left annihilator for $\ltimes$, that is, $(0) \ltimes \underline{u}=(0)$. A vector $\underline{w} \in \mathbb{A}^{n}$ is said to be prime if there exists no vector $\underline{v} \in \mathbb{A}^{m}$ and $\underline{v^{\prime}} \in \mathbb{A}^{m^{\prime}}$, with $n=m m^{\prime}$ such that $\underline{w}=\underline{v} \ltimes \underline{v}^{\prime}$. In general, the dendriform product of two vectors gives a grove. However, observe there are two unique ways to obtain a vector. The first one is to consider $(1) \dashv(1) \ltimes \underline{v}$, with $\underline{v}:=\underline{v}_{1} \vee(0)$ and the second one is to consider $(1) \vdash(1) \ltimes \underline{v}$, with $\underline{v}:=(0) \vee \underline{v}_{1}$. In the first case, we obtain $(1) \dashv(1) \ltimes \underline{v}=\underline{v}_{1} \vee\left(\underline{v}_{1} \vee(0)\right)$ and in the second case, $(1) \vdash(1) \ltimes \underline{v}=\left((0) \vee \underline{v}_{1}\right) \vee \underline{v}_{1}$. We summarize our discussion by the following proposition.

Proposition 2.19. Any vector of $\mathbb{A}^{2 n+1}$ is prime for the arithmetree just described. Whereas there exist $2 c_{n}$ nonprime vectors in $\mathbb{A}^{2 n+2}$. They are of the forms $((0) \vee \underline{v}) \vee \underline{v}$ and $\underline{v} \vee(\underline{v} \vee$ (0)), with $\underline{v} \in \mathbb{A}^{n}$.

Proposition 2.20 (right and left cancellations). Let $\underline{v} \in \mathbb{A}^{n}, \underline{u}, \underline{w} \in \mathbb{A}^{m}$. Then,

$$
\underline{v} \ltimes \underline{u}=\underline{v} \ltimes \underline{w} \Longleftrightarrow \underline{u}=\underline{w}, \quad \underline{u} \ltimes \underline{u}=\underline{w} \ltimes \underline{w} \Longleftrightarrow \underline{u}=\underline{w}, \quad \underline{u} \ltimes \underline{v}=\underline{w} \ltimes \underline{v} \Longleftrightarrow \underline{u}=\underline{w} .
$$

Proof. The first claim is obtained by observing that the first operation appearing in $\omega_{\underline{v}}((1))$ is either $\vdash$ or $\dashv$. Therefore, in both cases, the vectors composing the groves $\underline{v} \ltimes \underline{u}$ and $\underline{v} \ltimes \underline{w}$ will start with $(\underline{u}, \ldots)$, respectively, with $(\underline{w}, \ldots)$. The same remark applies also for the second claim. To complete the proof, observe that the dendriform multiplication acting on the right-hand side is the unique dendriform automorphism which maps the generator $X^{(1)}$ to $X^{\underline{v}}$ in $K\left[\mathbf{A}^{\bullet}\right]$. 
Proposition 2.21. Let $\underline{u}$ be a name of a binary tree. Then, $\Phi_{\underline{u}}((1))$ can be obtained from $\omega_{\underline{u}}((1))$ by replacing the symbols $\vdash$ by + , and $\dashv$ by $+\ldots$. We name the middle term the vector so obtained. If a grove is not prime for the dendriform arithmetics, then its middle term will be not prime for the L-arithmetics.

Proof. Proceed by induction. It is true for $n=1,2,3$ (checked by hand). Observe that $\underline{u}_{l} \vdash(1) \dashv \underline{u}_{r}=\underline{u}_{l} \vee \underline{u}_{r}=\underline{u}_{l}+,(1)+\vee \underline{u}_{r}$. Therefore, $\omega_{\underline{u}_{l}}(1) \vdash(1) \dashv \omega_{\underline{u}_{r}}(1)$ gives $\Phi_{\underline{u}_{l}}(1)+$, $(1)+{ }_{\star} \omega_{\underline{u}_{r}}(1)$ by replacing the symbols $\vdash$ by + , and $\dashv$ by $+\ltimes$.

\section{Bijection between noncrossing partitions and binary trees}

In the following two sections, we show how to reformulate the framework of Speicher [15] on free probability by using the free dendriform algebra on $Y$. We recall a bijection between noncrossing partitions and binary trees. A noncrossing partition of the set $\{1,2,3, \ldots, n\}$ is a decomposition $\pi:=\left\{V_{1}, \ldots, V_{r}\right\}$ of $S$ into disjoint and nonempty sets $V_{i}$, called blocks, such that for all $1 \leq p_{1}, q_{1}, p_{2}, q_{2} \leq n$, the following does not occur: there exist $1 \leq p_{1}<q_{1}<p_{2}<q_{2}$ with $p_{1} \sim_{\pi} p_{2} \nsim_{\pi} q_{1} \sim_{\pi} q_{2}$, where for all $1 \leq p, q \leq n, p \sim_{\pi} q$ means that $p$ and $q$ belong to the same block of $\pi$. The set of noncrossing partitions made out of the elements $1,2,3, \ldots, n$ is denoted by $\mathrm{NC}(n)$. In low dimensions, these sets are

$$
\begin{aligned}
& 1 \quad 1212 \quad 123123123123123
\end{aligned}
$$

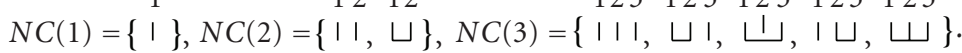

There is a natural poset structure given by the refinement order. In the sequel, an interval of a block $V$ is a sequence of numbers all linked to one another. Every block can be decomposed uniquely in several intervals. A bijection between noncrossing partitions and binary trees is determined by the following algorithm in 2 steps.

(1) Let $\tau \in Y_{n}$ be an $n$-tree, $n>0$. As the tree is planar and binary, the notion of left and right still has a meaning. Denote by 1 the root. This gives a frame denoted by $(1, R, L)$ where the axis $R$ (resp., $L$ ) is the line passing through 1 and identified with the rightmost (resp., leftmost) branch. Starting with the origin of the frame here $(1, L, R)$, that is, with 1 , increment of a unit all the $p>1$ branches linked to the axis $L$ giving $1,2,3 \ldots, p$. If there is no branch at the left-hand side of 1 , give 2 to the closest vertex at the right-hand side of 1 and reapply the algorithm.

(2) Once arrived at the vertex $p$. If there is a vertex to the right of $p$, give the number $p+1$ to it, and reapply the algorithm in the frame $(p+1, L, R)$ modelling now the subtree with root, the vertex $p+1$. If not, go to the vertex $p-1$ and reapply the algorithm at step (2).

Once all vertices of the tree are labeled, a unique noncrossing partition is obtained by the following trick. Put a vertical segment under each number $1,2, \ldots, n$ and link $p$ to $q>p$ if $q$ is the closest vertex at the right-hand side of $p$. One can view this partition as the "projection" (by abuse of language) parallel to the axis $L$ of all the branches of the trees on the axis $R$ in the frame $(1, L, R)$ (if the branches are all drawn either parallel to the axis $L$ or $R$ ). Here is the example $(1,9)(2,6,7)(3,4)(5)(8)(10)$ (written shortly like a 
permutation in disjoint cycles):

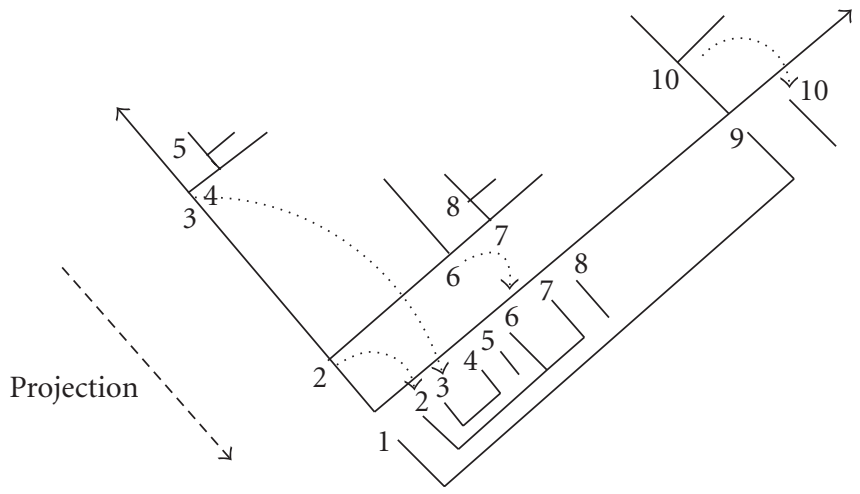

The construction of the inverse map is left to the reader. We therefore obtain a bijection between rooted binary trees and noncrossing partitions.

\section{Free probability}

Free probability has been introduced by Voiculescu. Later, a complementary point of view was given by Speicher [15], inspired by previous works of Rota [12]. We will focus on [15].

4.1. Action of arithmetree on $\mathscr{B}-\mathscr{B}$-bimodule and operads. In the sequel, $\mathscr{B}$ denotes a unital associative algebra (most of the time a unital $C^{*}$-algebra for applications) and $M$ is a $\mathscr{B}-\mathscr{B}$-bimodule. We denote by $\mathcal{M}^{\otimes_{\mathscr{B}} n}$ the space $\mathcal{M} \otimes_{\mathscr{B}} M \otimes_{\mathscr{B}} \cdots \otimes_{\mathscr{B}} M, n$ times and by convention $\mathcal{M}^{\otimes_{\mathscr{B}} 0}:=B$. By abuse of language and sometimes to ease notation, we will use equivalently trees and/or their names. One of the aims of this part is to describe the action of the space $K\left[\mathbb{Y}_{\infty}\right]$ - or equivalently $K\left[\mathbf{A}^{\bullet}\right]$ —equipped with its arithmetree onto the bimodule $M$.

4.1.1. $\mathrm{NCP}(\mathscr{B})$-operads. To introduce the action of binary trees in terms of noncrossing partitions, we will need the concept of noncrossing partitions operads, NCP( $\mathscr{B})$-operads for short.

Definition 4.1. Let $\mathscr{B}$ be an associative $K$-algebra. A NCP( $\mathscr{B})$-operad $\mathbb{P}$ (without unit) over a $\mathscr{B}-\mathscr{B}$-bimodule $\mathcal{M}$ is the data of a family of finite dimensional $K$-vector spaces $(\mathbb{P}(n))_{n>0}$, whose basis elements $\mu$ are $\mathscr{B}-\mathscr{B}$-bimodule $n$-ary operations with values in $\mathscr{B}$, that is, $\mu: \mathcal{M}^{\otimes_{\mathscr{B}}}{ }^{n} \rightarrow \mathscr{B}$, and equipped with a family of composition maps $\left(\left(\circ_{i}\right)_{i>0}\right)$ verifying the following relations.

(1) For all $\mu \in \mathbb{P}(m)$ and $\nu \in \mathbb{P}(n)$ and $1 \leq i \leq m+1, \mu \circ_{i} \nu \in \mathbb{P}(m+n)$.

(2) For all $\lambda \in \mathbb{P}(l), \mu \in \mathbb{P}(m)$, and $\nu \in \mathbb{P}(n)$,

$$
\begin{gathered}
\left(\lambda \circ_{i} \mu\right) \circ_{j+m} \nu=\left(\lambda \circ_{j} \nu\right) \circ_{i} \mu, \quad 1 \leq i \leq j \leq l+1, \\
\lambda \circ_{i}\left(\mu \circ_{j} \nu\right)=\left(\lambda \circ_{i} \mu\right) \circ_{i+j-1} \nu, \quad 1 \leq i \leq l+1,1 \leq j \leq m+1 .
\end{gathered}
$$


Remark 4.2. This concept is inspired from [15] but is different, though similar, from the definition of a regular $K$-linear operad over a vector space $M$. Indeed, take $\mathscr{B}$ as the ground field. In the usual operad theory, the basis elements $\mu$ are $n$-ary operations with values in $M$, that is, $\mu: M^{\otimes_{\mathscr{B}}}{ }^{n} \rightarrow \mathcal{M}$, and not with values in the ground field. This entails a slight modification of the usual axioms of an operad over a vector space $M$ presented just above.

A noncrossing partition $\mu \in \mathrm{NC}(n)$ is said to be decorated by a set $\mathrm{Col}$ if a unique color of $\mathrm{Col}$ is associated with each interval composing it. Observe that decorated noncrossing partitions give special decorated binary trees, that is, binary trees whose all vertices of a SW-NE-branch have the same color. We now give an example of such $\mathrm{NCP}(\mathscr{B})$-operad by mixing results in Section 2 on the free dendriform algebra on one generator and ideas developed from noncommutative probability.

4.1.2. NCP( $\mathscr{B})$-operads and dendriform structure on $\mathscr{B}-\mathscr{B}$-bimodules. Let $\mathcal{M}$ be a $\mathscr{B}-$ $\mathscr{B}$-bimodule. The dendriform algebra structure over $K\left[\mathbf{A}_{*}^{*}\right]$ induces a dendriform algebra structure on the following $K$-vector space: $\operatorname{Dend}_{\mathscr{B}}(\mathcal{M}):=\bigoplus_{n \geq 1} K\left[\mathbf{A}^{n}\right] \otimes M^{\otimes_{\mathscr{B}}}{ }^{n}$, by declaring that

$$
\begin{aligned}
& X^{\underline{v}} \otimes \kappa \prec X^{\underline{w}} \otimes \kappa^{\prime}:=X^{\underline{v}} \prec X^{\underline{w}} \otimes \kappa \kappa^{\prime}=X^{\underline{v}-\underline{w}} \otimes \kappa \kappa^{\prime}, \\
& X^{\underline{v}} \otimes \kappa \succ X^{\underline{w}} \otimes \kappa^{\prime}:=X^{\underline{v}} \succ X^{\underline{w}} \otimes \kappa \kappa^{\prime}=X^{\underline{v}-\underline{w}} \otimes \kappa \kappa^{\prime},
\end{aligned}
$$

for any tensor $\kappa \in \mathcal{M}^{\otimes_{\mathscr{B}}}{ }^{n}$ and $\kappa^{\prime} \in \mathcal{M}^{\otimes_{\mathscr{B}}} n^{\prime}$. Inspired by [15], we define a family of $n$-ary operations, $f^{(n)}: \mathcal{M}^{\otimes_{\mathscr{B}}}{ }^{n} \rightarrow \mathscr{B}$, which are $\mathscr{B}$ - $\mathscr{B}$-bimodule maps, that is, $f^{(n)}\left(b a_{1} \otimes_{\mathscr{B}} a_{2} \otimes_{\mathscr{B}}\right.$ $\left.\cdots \otimes_{\mathscr{B}} a_{n} b^{\prime}\right):=b f^{(n)}\left(a_{1} \otimes_{\mathscr{B}} a_{2} \otimes_{\mathscr{B}} \cdots \otimes_{\mathscr{B}} a_{n}\right) b^{\prime}$, for all $n>0$ and $b, b^{\prime} \in \mathscr{B}$ and $a_{1}, \ldots, a_{n} \in$ M. With each family $\left(f^{(n)}\right)_{n \geq 1}$, we associate the following operator valued function $\hat{f}$, acting on the whole $\operatorname{Dend}_{\Re}(\mathcal{M})$ :

$$
\begin{gathered}
\hat{f}:=\left(f^{(n)}\right)_{n \geq 1}: \operatorname{Dend}_{\mathscr{B}}(\mathcal{M}) \longrightarrow \mathscr{B}, \\
\left(X^{\underline{v}} \otimes a_{1} \otimes_{\mathscr{B}} a_{2} \otimes_{\mathscr{B}} \cdots \otimes_{\mathscr{R}} a_{n}\right) \longmapsto \hat{f}\left(X^{\underline{v}} \otimes a_{1} \otimes_{\mathscr{B}} a_{2} \otimes_{\mathscr{B}} \cdots \otimes_{\mathscr{B}} a_{n}\right),
\end{gathered}
$$

and defined via the following recursive prescription. With any monomial $X^{\underline{v}}$, a unique noncrossing partition $\operatorname{Pr}(\underline{v})$ is associated, constructed from the algorithm described in the previous section. Identify this partition to the tensor $a_{1} \otimes_{\mathscr{B}} a_{2} \otimes_{\mathscr{B}} \cdots \otimes_{\mathscr{B}} a_{n}$. Localize the most nested block of length $p \leq n$ and apply the $p$-ary operations, giving thus an operator in $\mathscr{B}$. Then, reapply this procedure. In the sequel, we will write

$$
\begin{aligned}
\left(X^{\underline{v}} \otimes a_{1} \otimes_{\mathscr{B}} a_{2} \otimes_{\mathscr{B}} \cdots \otimes_{\mathscr{B}} a_{n}\right) & \longmapsto \hat{f}\left(X^{\underline{v}} \otimes a_{1} \otimes_{\mathscr{B}} a_{2} \otimes_{\mathscr{A}} \cdots \otimes_{\mathscr{B}} a_{n}\right) \\
& :=\hat{f}\left(\operatorname{Pr}(\underline{v}) \leadsto\left(a_{1} \otimes_{\mathscr{B}} a_{2} \otimes_{\mathscr{B}} \cdots \otimes_{\mathscr{B}} a_{n}\right)\right),
\end{aligned}
$$

to denote that action of the noncrossing partition $\operatorname{Pr}(\underline{v})$. The following examples will be better than a fastidious description. Here are three examples (recall that $a \otimes_{\mathscr{B}} b a^{\prime}=$ $a b \otimes_{\mathscr{B}} a^{\prime}$, for $\left.b \in \mathscr{B}\right)$.
(1) $\hat{f}(X$ $\left.\otimes a_{1} \otimes_{\mathscr{乃}} a_{2} \otimes_{\mathscr{乃}} a_{3}\right)=f^{(2)}\left(a_{1} \otimes_{\mathscr{B}} f^{(1)}\left(a_{2}\right) \otimes_{\mathscr{乃}} a_{3}\right)=f^{(2)}\left(a_{1} f^{(1)}\left(a_{2}\right) \otimes_{\mathscr{R}} a_{3}\right)$.
(2) $\hat{f}(X$ $\left.\otimes a_{1} \otimes_{\mathscr{R}} a_{2} \otimes_{\mathscr{R}} a_{3}\right)=f^{(2)}\left(a_{1} \otimes_{\mathscr{R}} a_{2}\right) \otimes_{\mathscr{R}} f^{(1)}\left(a_{3}\right)=f^{(2)}\left(a_{1} \otimes_{\mathscr{R}} a_{2}\right) f^{(1)}\left(a_{3}\right)$. 
14 Free dendriform algebras. Part I. A parenthesis setting

(3) Let $\operatorname{Pr}(\underline{v})=(1,9)(2,6,7)(3,4)(5)(8)(10)$ be the noncrossing partition represented in Section 3 and get

$$
\begin{aligned}
\hat{f}\left(X^{\underline{v}} \otimes\right. & \left.a_{1} \otimes_{\mathscr{B}} a_{2} \otimes_{\mathscr{B}} \cdots \otimes_{\mathscr{B}} a_{10}\right)=\hat{f}\left(\operatorname{Pr}(\underline{v}) \sim\left(a_{1} \otimes_{\mathscr{B}} a_{2} \otimes_{\mathscr{B}} \cdots \otimes_{\mathscr{B}} a_{10}\right)\right) \\
= & f^{(2)}\left(a_{1} \otimes_{\mathscr{B}} f^{(3)}\left(a_{2} \otimes_{\mathscr{B}} f^{(2)}\left(a_{3} \otimes_{\mathscr{B}} a_{4}\right) \otimes_{\mathscr{B}} f^{(1)}\left(a_{5}\right) \otimes_{\mathscr{B}} a_{6} \otimes_{\mathscr{B}} a_{7}\right)\right. \\
& \left.\otimes_{\mathscr{R}} f^{(1)}\left(a_{8}\right) \otimes_{\mathscr{B}} a_{9}\right) \otimes_{\mathscr{B}} f^{(1)}\left(a_{10}\right)
\end{aligned}
$$

and obtain $f^{(2)}\left(a_{1} f^{(3)}\left(a_{2} f^{(2)}\left(a_{3} \otimes_{\mathscr{R}} a_{4}\right) f^{(1)}\left(a_{5}\right) \otimes_{\mathscr{R}} a_{6} \otimes_{\mathscr{R}} a_{7}\right) \otimes_{\mathscr{B}} f^{(1)}\left(a_{8}\right) a_{9}\right) f^{(1)}$ $\left(a_{10}\right)$.

Remark 4.3. Proceeding that way, observe that $f^{(n)}\left(a_{1} \otimes_{\mathscr{R}} a_{2} \otimes_{\mathscr{R}} \cdots \otimes_{\mathscr{R}} a_{n}\right)$ and the action of the maximal element $\mathbf{1}_{n}$ of $\mathrm{NC}(n)$ on the $n$-tensor, that is, $f^{(n)}\left(\mathbf{1}_{n} \rightsquigarrow a_{1} \otimes_{\mathscr{A}} a_{2} \otimes_{\mathscr{A}}\right.$ $\left.\cdots \otimes_{\mathscr{R}} a_{n}\right)$, coincide.

Remark 4.4. We can slightly reformulate this framework using the concept of $\mathrm{NCP}(\mathscr{B})$ operad. Let $\mathrm{Col}:=\left\{f^{(n)}, n>0\right\}$ be the color set made out of the $n$-ary operations $f^{(n)}$. Observe that with each noncrossing partition, a unique decorated noncrossing partition can be associated. Introduce the object $\mathbb{P}[\hat{f}]$ made out of a family of the $K$-vector spaces $(\mathbb{P}[\hat{f}](n))_{n>0}$ and the family of composition $\left(o_{i}\right)_{i>0}$ defined by induction as follows. The $K$-vector space $\mathbb{P}[\hat{f}](1)$ is spanned by $f^{(1)}$ and $\mathbb{P}[\hat{f}](p)$ by the elements $f^{(p)}$ and the $\mu \circ_{i} \nu$ for all $1 \leq i \leq m+1$, with $p=n+m, \mu \in \mathbb{P}[\hat{f}](m)$ and $\nu \in \mathbb{P}[\hat{f}](n)$ such that

$$
\begin{aligned}
& \mu \circ_{i} \nu\left(a_{1} \otimes_{\mathscr{R}} \cdots \otimes_{\mathscr{R}} a_{n+m}\right) \\
& \quad:=\left(a_{1} \otimes_{\mathscr{B}} \cdots \otimes_{\mathscr{R}} a_{i-1} \otimes_{\mathscr{R}} \nu\left(a_{i} \otimes_{\mathscr{R}} \cdots \otimes_{\mathscr{B}} a_{i+n-1}\right) \otimes_{\mathscr{B}} a_{i+n} \otimes_{\mathscr{B}} \cdots \otimes_{\mathscr{B}} a_{m+n}\right),
\end{aligned}
$$

for all $a_{1}, \ldots, a_{n} \in \mathcal{M}$ and not in $\mathscr{B}$. From a noncrossing partition, one can easily write its action on tensor elements in terms of composition maps. The following example will fix ideas.

Example 4.5. Consider again $\operatorname{Pr}(\underline{v}):=(1,9)(2,6,7)(3,4)(5)(8)(10)$, the noncrossing partition represented in Section 3. Read the partition from left to right. Take the first encountered interval, say, with $p$ elements (here $\{1,9\}$ and $p:=2$ ) and (thus) starting with 1 . Take the second encountered interval, starting with say $n$, and with say $q$ elements (here $\{2,6,7\}$ and $q:=3$ ) and write $f^{(p)} \circ_{n} f^{(q)} \cdots$. Reapply the algorithm. We obtain

$$
\begin{aligned}
& \hat{f}\left(X^{\underline{v}} \otimes a_{1} \otimes_{\mathscr{B}} a_{2} \otimes_{\mathscr{B}} \cdots \otimes_{\mathscr{B}} a_{10}\right) \\
& \quad:=f^{(2)} \circ_{2} f^{(3)} \circ_{3} f^{(2)} \circ_{5} f^{(1)} \circ_{8} f^{(1)} \circ_{10} f^{(1)}\left(a_{1} \otimes_{\mathscr{B}} a_{2} \otimes_{\mathscr{B}} \cdots \otimes_{\mathscr{B}} a_{10}\right) .
\end{aligned}
$$

The following theorem summarizes the previous discussion.

Theorem 4.6. Let $\mathscr{B}$ be an associative algebra and let $\mathcal{M}$ be a $\mathscr{B}-\mathscr{B}$-bimodule. Let $\hat{f}:=$ $\left(f^{(n)}: \operatorname{Dend}_{\mathscr{B}}(\mathcal{M}) \rightarrow \mathscr{B}\right)_{n>0}$ be a family of $n$-ary $\mathscr{B}-\mathscr{B}$-bimodule operations. Then, (Dend $\left.\mathscr{B}_{\beta}(\mathcal{M}), \hat{f}\right)$ induces a $\mathrm{NCP}(\mathscr{B})$-operad, $\mathbb{P}[\hat{f}]$, over the $B-B$-bimodule $\mathcal{M}$. 
4.2. Cumulants and moments in free probability. We recall some properties of free probability [15]. Fix $B$, a unital associative algebra. Let $(\mathcal{M}, \phi)$ be a noncommutative probability space, that is, a $B-B$-bimodule $M$ endowed with a unital associative algebra structure equipped with a $B-B$-bimodule map $\phi: M \rightarrow B$ such that $\phi(1)=1$, that is, $\phi(b)=b$,

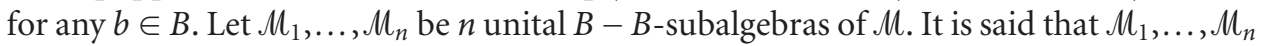
are stochastically free if $\phi\left(a_{1} \cdots a_{n}\right)=0$ under the following conditions. For all $1 \leq i \leq$ $n, \phi\left(a_{i}\right)=0$ and for $a_{1} \in \mathcal{M}_{\epsilon_{1}}, \ldots, a_{n} \in \mathcal{M}_{\epsilon_{n}}, \epsilon_{1} \neq \epsilon_{2}, \epsilon_{2} \neq \epsilon_{3}, \ldots, \epsilon_{n-1} \neq \epsilon_{n}$. It has been shown by Speicher, that this definition can be reformulated in terms of noncrossing partitions equipped with the refinement order. For that, he introduced in [15], the set $\bigcup_{n>1} \mathrm{NC}(n) \times \mathcal{M}^{\otimes_{\mathscr{B}}}{ }^{n}$ and a family of functions $\hat{\phi}=\left(\phi^{(n)}\right)_{n>1}: \bigcup_{n>1} \mathrm{NC}(n) \times \mathcal{M}^{\otimes_{\mathscr{B}}}{ }^{n} \rightarrow B$ and defined $\hat{\phi}(\pi)\left(a_{1} \otimes_{\mathscr{R}} \cdots \otimes_{\mathscr{R}} a_{n}\right)$, where $\pi$ is a noncrossing partition, as explained in the previous section. The idea is to replace the object $\bigcup_{n>1} \mathrm{NC}(n) \times \mathcal{M}^{\otimes_{\mathscr{B}}}{ }^{n}$ by a much more structured one, that is, $\operatorname{Dend}_{\mathscr{B}}(\mathcal{M})$ equipped with the $\operatorname{NCP}(\mathscr{B})$-operad $\mathbb{P}[\hat{\phi}]$. We are now able to reformulate the major result of Speicher [15]. Recall that a moment function [15] $\phi$ is defined by $\phi^{(1)}(1)=1$ and by $(n>1)$,

$\phi^{(n-1)}\left(a_{1} \otimes_{\mathscr{B}} \cdots \otimes_{\mathscr{B}} a_{p} a_{p+1} \otimes_{\mathscr{R}} \cdots \otimes_{\mathscr{\beta}} a_{n}\right)=\phi^{(n)}\left(a_{1} \otimes_{\mathscr{B}} \cdots \otimes_{\mathscr{R}} a_{p} \otimes_{\mathscr{R}} a_{p+1} \otimes_{\mathscr{R}} \cdots \otimes_{\mathscr{B}} a_{n}\right)$.

In this case, one can choose $\phi^{(n)}\left(a_{1} \otimes_{\mathscr{R}} \cdots \otimes_{\mathscr{R}} a_{n}\right):=\phi^{(1)}\left(a_{1} a_{2} \cdots a_{n}\right)$. In our framework, Speicher showed also that the cumulant function $\hat{C}$ obtained by convolution of $\hat{\phi}$ with the Zeta function associated with the refinement order of the noncrossing partitions is still a map from $\operatorname{Dend}_{\mathscr{B}}(\mathcal{M})$ to $B$. We now reformulate the result of Speicher [15].

Theorem 4.7 [15]. Fix B, a unital associative algebra. Let $(M, \phi)$ be a noncommutative probability space and $\phi$ a moment function. Let $M_{1}, \ldots, M_{n}$ be $n$ unital $B-B$-subalgebras of M. Consider the set $I:=\left\{X^{\underline{n}} \otimes a_{1} \otimes_{\mathscr{B}} \cdots \otimes_{\mathscr{B}} a_{n} \in \mathcal{M}\right.$; for all $n>1$; such that $\exists i, j a_{i} \in \mathcal{M}_{\epsilon_{i}}$, $a_{j} \in M_{\epsilon_{j}}$, and $\left.\epsilon_{i} \neq \epsilon_{j}\right\}$. Then, $M_{1}, \ldots, M_{n}$ are stochastically free if and only if $I \subseteq \operatorname{ker} \hat{C}$, where $\hat{C}: \operatorname{Dend}_{\mathscr{B}}(M) \rightarrow B$ is the cumulant function associated with $\hat{\phi}$ via the convolution with the Zeta function with respect to the refinement order.

\section{Acknowledgments}

The author would like to thank Michael Schürmann, Uwe Franz, Rolf Gohm, and Stefanie Zeidler for their very warm hospitality during his stay at the Institut für Mathematik und Informatik, Greifswald, Germany, where this paper has been written. The work is supported by the European Commission HPRN CTN2002 00279, RTN QPApplications.

\section{References}

[1] V. Ginzburg and M. Kapranov, Koszul duality for operads, Duke Mathematical Journal 76 (1994), no. 1, 203-272.

[2] Ph. Leroux, An algebraic framework of weighted directed graphs, International Journal of Mathematics and Mathematical Sciences 2003 (2003), no. 58, 3657-3678.

[3] Algebra 32 (2004), no. 8, 2949-2967. 
[4] J.-L. Loday, La renaissance des opérades, Astérisque 237 (1996), 47-74, Exp. No. 792, 3.

[5] _ Dialgebras, Dialgebras and Related Operads, Lecture Notes in Math., vol. 1763, Springer, Berlin, 2001, pp. 7-66.

[6] , Arithmetree, Journal of Algebra 258 (2002), no. 1, 275-309.

[7] J.-L. Loday and M. O. Ronco, Hopf algebra of the planar binary trees, Advances in Mathematics 139 (1998), no. 2, 293-309.

[8] - Order structure on the algebra of permutations and of planar binary trees, Journal of Algebraic Combinatorics 15 (2002), no. 3, 253-270.

[9] C. Malvenuto and C. Reutenauer, Duality between quasi-symmetric functions and the Solomon descent algebra, Journal of Algebra 177 (1995), no. 3, 967-982.

[10] M. Markl, S. Shnider, and J. Stasheff, Operads in Algebra, Topology and Physics, Mathematical Surveys and Monographs, vol. 96, American Mathematical Society, Rhode Island, 2002.

[11] T. Pirashvili, Sets with two associative operations, Central European Journal of Mathematics 1 (2003), no. 2, 169-183.

[12] G.-C. Rota (ed.), Finite Operator Calculus, Academic Press, New York, 1975.

[13] G.-C. Rota, Gian-Carlo Rota on Combinatorics. Introductory Papers and Commentaries, edited by J. P. S. Kung, Contemporary Mathematicians, Birkhäuser Boston, Massachusetts, 1995.

[14] B. E. Sagan, A generalization of Rota's NBC theorem, Advances in Mathematics 111 (1995), no. 2, 195-207.

[15] R. Speicher, Combinatorial theory of the free product with amalgamation and operator-valued free probability theory (Habilitationsschrift), Memoirs of the American Mathematical Society 132 (1998), no. 627, $x+88$.

Philippe Leroux: Institut für Mathematik und Informatik, Ernst-Moritz-Arndt-Universität, Jahnstraße 15a, 17487 Greifswald, Germany

E-mail addresses: ph_ler_math@yahoo.com; leroux@uni-gerifswald.de 


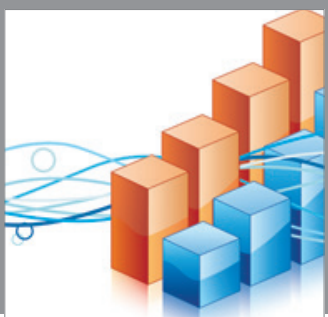

Advances in

Operations Research

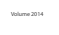

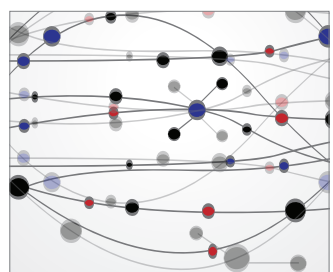

\section{The Scientific} World Journal
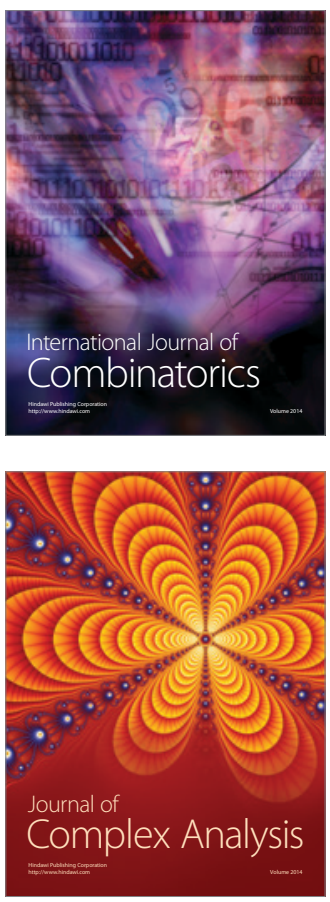

International Journal of

Mathematics and

Mathematical

Sciences
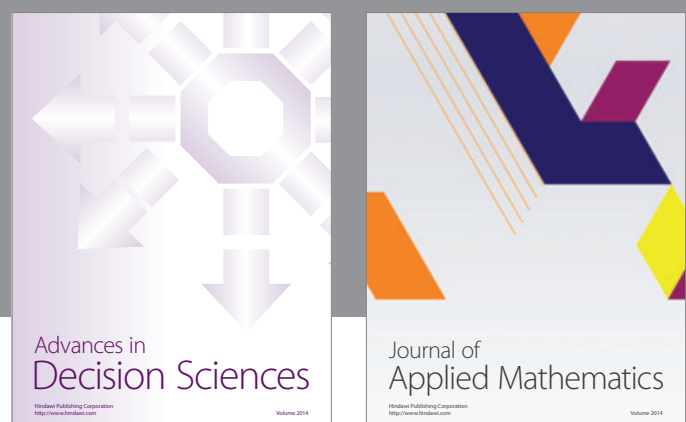

Journal of

Applied Mathematics
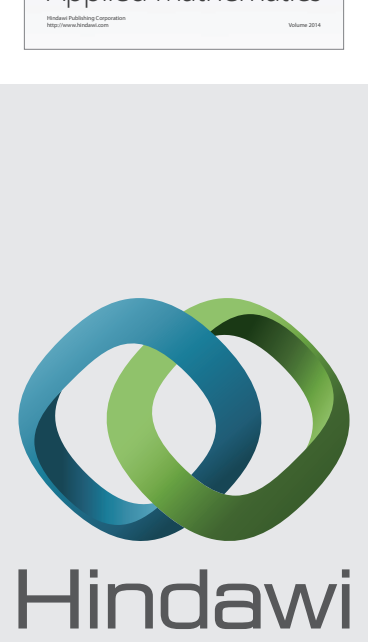

Submit your manuscripts at http://www.hindawi.com
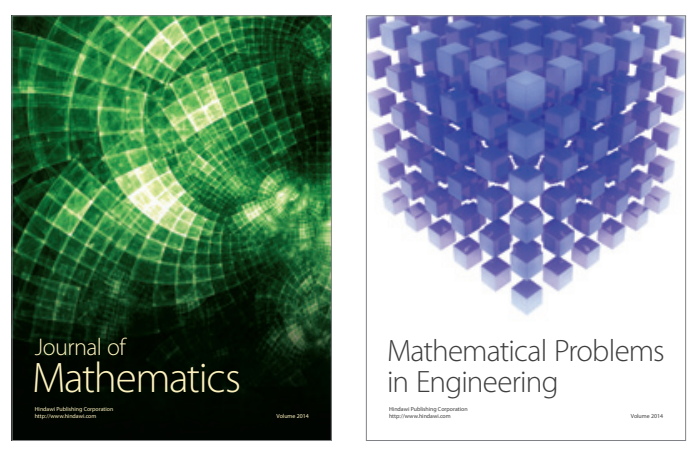

Mathematical Problems in Engineering
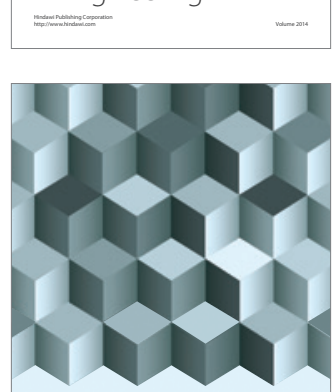

Journal of

Function Spaces
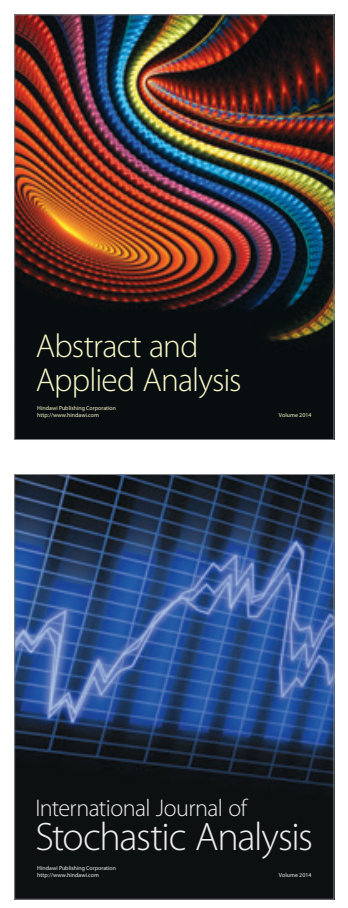

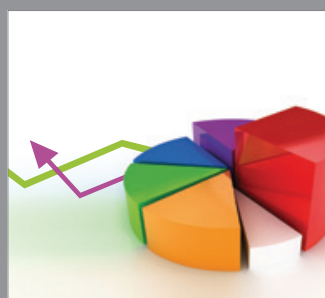

ournal of

Probability and Statistics

Promensencen
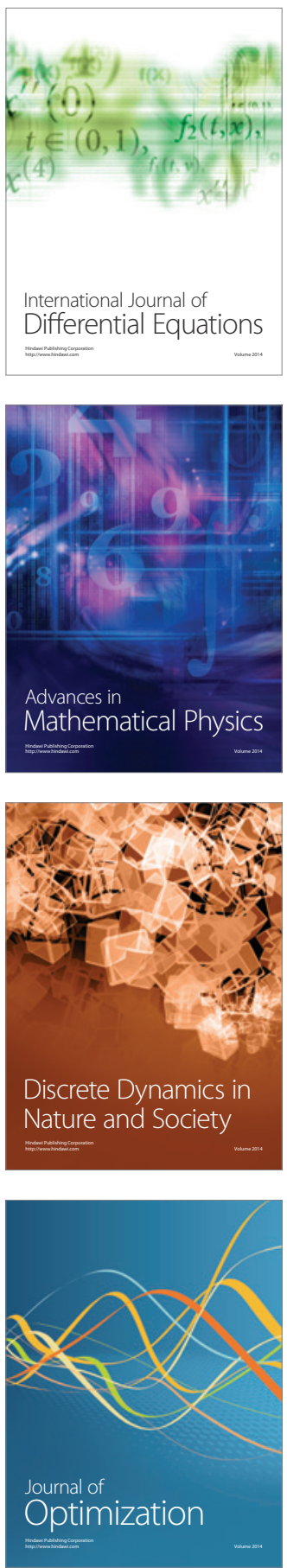